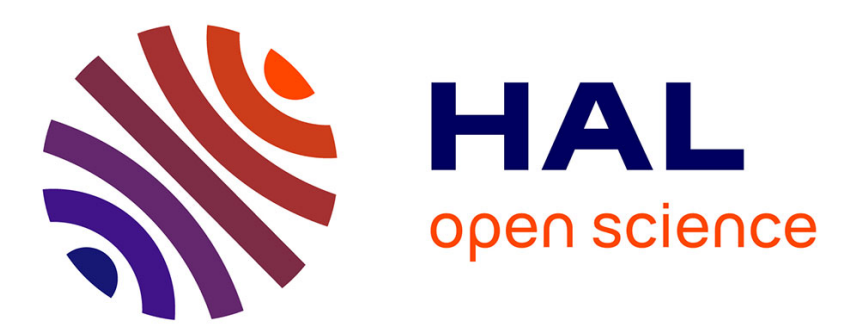

\title{
Corrections to scaling and phenomenological renormalization for 2-dimensional percolation and lattice animal problems
}

B. Derrida, D. Stauffer

\section{- To cite this version:}

B. Derrida, D. Stauffer. Corrections to scaling and phenomenological renormalization for 2dimensional percolation and lattice animal problems. Journal de Physique, 1985, 46 (10), pp.16231630. 10.1051/jphys:0198500460100162300 . jpa-00210110

HAL Id: jpa-00210110

https://hal.science/jpa-00210110

Submitted on 1 Jan 1985

HAL is a multi-disciplinary open access archive for the deposit and dissemination of scientific research documents, whether they are published or not. The documents may come from teaching and research institutions in France or abroad, or from public or private research centers.
L'archive ouverte pluridisciplinaire HAL, est destinée au dépôt et à la diffusion de documents scientifiques de niveau recherche, publiés ou non, émanant des établissements d'enseignement et de recherche français ou étrangers, des laboratoires publics ou privés. 


\title{
Corrections to scaling and phenomenological renormalization for 2-dimensional percolation and lattice animal problems
}

\author{
B. Derrida and D. Stauffer $\left({ }^{*}\right)$ \\ Service de Physique Théorique, CEN-Saclay, 91191 Gif sur Yvette Cedex, France
}

(Reçu le 22 mars 1985, accepté le 3 juin 1985)

\begin{abstract}
Résumé. - Nous continuons et améliorons l'approche par matrice de transfert de Derrida et de Seze en tenant compte de deux manières différentes de la correction dominante. Nous obtenons pour le seuil de percolation de site sur le réseau carré $p_{\mathrm{c}}=0,59274 \pm 0,00010$, pour l'exposant qui caractérise la taille des animaux $0,64075 \pm$ 0,00015 et pour la fugacité critique $0,246150 \pm 0,000010$ sur le réseau carré et $0,192925 \pm 0,000010$ sur le réseau triangulaire. Ces résultats sont en accord, et parfois plus précis, que les meilleures estimations connues.
\end{abstract}

\begin{abstract}
We continue and improve the transfer matrix approach of Derrida and de Seze by incorporating in two different ways the leading corrections to the asymptotic behaviour for wide strips. We find for the site percolation threshold in the square lattice $p_{c}=0.59274 \pm 0.00010$, for the radius exponent of lattice animals $0.64075 \pm 0.00015$, and for the inverse growth factor or critical fugacity $0.246150 \pm 0.000010$ in the square lattice and $0.192925 \pm 0.000010$ in the triangular lattice. These results are consistent with, and sometimes more accurate than, the best estimates published before.
\end{abstract}

\section{Introduction.}

In the numerical analysis of critical phenomena one may ask two types of questions : what are the best results for a quantity which is not known exactly; and what is the best method to evaluate this quantity. The phenomenological renormalization or transfer matrix approach has proven to be an accurate way of calculating phase diagrams and critical exponents for many two-dimensional models of statistical mechanics [1-3]. It consists in calculating the physical properties (for example the correlation length $\xi_{n}$ or the susceptibility $\chi_{n}$ ) of a strip of width $n$ with an arbitrarily high accuracy using a transfer matrix technique. Then from the knowledge of $\xi_{n}$ or $\chi_{n}$ one can estimate the critical point $T_{\mathrm{c}}$ of the infinite lattice, or its critical exponents $v$ or $\gamma$ by assuming that $\xi_{n}$ and $\chi_{n}$ asymptotically satisfy a finite-size scaling law :

$$
\begin{aligned}
& \xi_{n}=n F\left(n^{1 / v}\left(T-T_{\mathrm{c}}\right)\right) \\
& \chi_{n}=n^{\gamma / v} G\left(n^{1 / v}\left(T-T_{\mathrm{c}}\right)\right) .
\end{aligned}
$$

\footnotetext{
* Present and permanent address : Institute of Theoretical Physics, Cologne University, 5000 Köln 41, F.R.G.
}

The usual way of calculating the critical temperature $T_{\mathrm{c}}$ is to find the solution $T_{\mathrm{c}}^{(n)}$ of the fixed-point equation:

$$
\frac{1}{n} \xi_{n}\left(T_{c}^{(n)}\right)=\frac{1}{n-1} \xi_{n-1}\left(T_{c}^{(n)}\right) .
$$

Once $T_{c}^{(n)}$ is known, one can estimate the critical exponent $v$ of the correlation length $\xi \propto\left(T-T_{\mathrm{c}}\right)^{-v}$ from the sequence $v_{n}$ calculated by :

$$
1+1 / v_{n}=\log \left[\frac{\mathrm{d} \xi_{n}}{\mathrm{~d} T} / \frac{\mathrm{d} \xi_{n-1}}{\mathrm{~d} T}\right] / \log \frac{n}{n-1}
$$

where the derivatives in (4) are calculated at $T=$ $T_{\mathrm{c}}^{(n)}$.

Since the finite size scaling formula (1) is assumed to be valid only for large $n$ and small $T-T_{\mathrm{c}}$ one cannot expect equations $(3,4)$ to give the exact values of $T_{\mathrm{c}}$ and $v$. On the other hand, the successive estimates $T_{\mathrm{c}}^{(n)}$ and $v_{n}$ will approach $T_{\mathrm{c}}$ and $v$ if $n \rightarrow \infty$.

For the few models which can be solved exactly, like the two-dimensional Ising model or the $d$-dimensional spherical model, the analytic expression for $\xi_{n}$ is known for all $n$ and one can study analytically how $T_{\mathrm{c}}^{(n)}$ and $v_{n}$ approach their limits $T_{\mathrm{c}}$ 
and $v$. For the two-dimensional Ising model one can show [3] that $T_{c}^{(n)}$ converges as $n^{-3}$ and $v_{n}$ as $n^{-2}$ for large $n$.

For the models which have not been solved exactly for arbitrary large $n$, the width $n$ of the strips for which $\xi_{n}$ can be calculated is limited by the size of the transfer matrix, which for an exact evaluation increases exponentially with $n$. Presently available computers thus typically reach a width of order ten for twodimensional geometric problems like self-avoiding walks, percolation and lattice animals. Therefore one is able to use equations $(3,4)$ only for $n$ between 2 and about 10 and ends up with a sequence of a few numbers that one would like to extrapolate to the limit $n \rightarrow \infty$. The convergence is expected to be a power law [3] due to corrections to scaling [4-6]. In the present paper we describe two approaches to incorporate these correction terms in the phenomenological renormalization and try to take into account carefully these corrections to scaling effects. Our goal is to obtain estimates as accurate as possible for the critical point, and the critical exponents of twodimensional models : percolation and lattice animals.

\section{Methods of extrapolation.}

Derrida and de Seze looked at the whole sequence of data $v_{n}$ and $T_{c}^{(n)}$ and found which common correction exponent $\omega$ in [3] :

$$
\begin{gathered}
v_{n}-v \propto n^{-\omega} \\
T_{c}^{(n)}-T_{c} \propto n^{-\omega-1 / v}
\end{gathered}
$$

Table I. - Percolation thresholds $p_{\mathrm{c}}$ or critical fugacities $x_{\mathrm{c}}$, exponents $v$ and $\eta$, and matrix size $S_{n}$, obtained from equations $(3,4)$, for a posteriori analysis, equation (7), of percolation and lattice animals. Minor inaccuracies in ref. [3] are corrected.

Percolation, geometry A

$\begin{array}{rc}n & n-1 \\ 2 & 1 \\ 3 & 2 \\ 4 & 3 \\ 5 & 4 \\ 6 & 5 \\ 7 & 6 \\ 8 & 7 \\ 9 & 8 \\ 10 & 9 \\ 11 & 10 \\ \infty & \end{array}$

$p_{c}$
0.733891857
0.582141884
0.590956749
0.588696015
0.589917123
0.590682119
0.591223649
0.591593437
0.591852804

0.5927

$v$
1.5604
1.4932
1.4718
1.4139
1.3880
1.3717
1.3616
1.3550
1.3504

$4 / 3$

$\begin{array}{cr}n / \pi \xi_{n} & S_{n} \\ 0.098483 & 2 \\ 0.227139 & 3 \\ 0.215396 & 5 \\ 0.219018 & 8 \\ 0.216723 & 16 \\ 0.215093 & 29 \\ 0.213807 & 62 \\ 0.212843 & 129 \\ & 293 \\ & 666\end{array}$

Percolation, geometry B

$\begin{array}{cc}n & n-1 \\ 2 & 1 \\ 3 & 2 \\ 4 & 3 \\ 5 & 4 \\ 6 & 5 \\ 7 & 6 \\ 8 & 7 \\ \infty & \end{array}$

$p_{c}$
0.381966011
0.557309978
0.584660388
0.589195538
0.590799289
0.591537723
0.591934685
$\simeq 0.5928$

$v$
1.6353
1.2853
1.3283
1.3368
1.3381
1.3379
1.3375
$4 / 3$

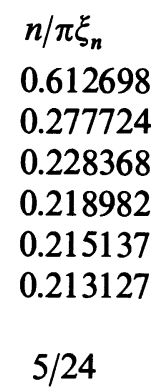

Percolation, geometry $\mathrm{C}$

$\begin{array}{rc}n & n-1 \\ 2 & 1 \\ 3 & 2 \\ 4 & 3 \\ 5 & 4 \\ 6 & 5 \\ 7 & 6 \\ 8 & 7 \\ 2 & 8 \\ 10 & 9 \\ \infty & \end{array}$

$\begin{array}{cc}p_{\mathrm{c}} & v \\ 0.381966011 & 1.6353 \\ 0.499158967 & 1.3722 \\ 0.497269222 & 1.3701 \\ 0.497879048 & 1.3549 \\ 0.498580100 & 1.3470 \\ 0.499042093 & 1.3427 \\ 0.499330781 & 1.3402 \\ 0.499515518 & 1.3386 \\ 0.499638152 & 1.3375 \\ 1 / 2 & 4 / 3\end{array}$

$\begin{array}{lr}n / \pi \xi_{n} & S_{n} \\ 0.353741 & 1 \\ 0.212302 & 2 \\ 0.214808 & 4 \\ 0.213808 & 7 \\ 0.212458 & 14 \\ 0.211443 & 27 \\ 0.210733 & 58 \\ 0.210233 & 124 \\ & 284 \\ 5 / 24 & \end{array}$


TABLE I (continued).

Lattice Animals, geometry A

\begin{tabular}{rcc}
$n$ & $n-1$ & \multicolumn{1}{c}{$x_{\mathrm{c}}$} \\
2 & 1 & 0.381966011 \\
3 & 2 & 0.252645801 \\
4 & 3 & 0.247567244 \\
5 & 4 & 0.245861670 \\
6 & 5 & 0.245788505 \\
7 & 6 & 0.245872936 \\
8 & 7 & 0.245951150 \\
9 & 8 & 0.246006101 \\
10 & 9 & 0.246043597 \\
11 & 10 & 0.246069536 \\
$\infty$ & & $\simeq 0.24613$
\end{tabular}

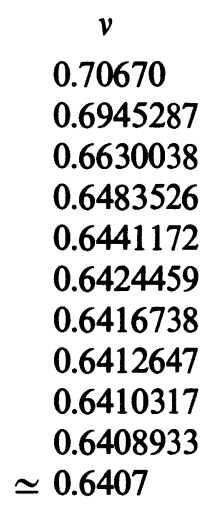

Lattice Animals, geometry B

$\begin{array}{cc}n & n-1 \\ 2 & 1 \\ 3 & 2 \\ 4 & 3 \\ 5 & 4 \\ 6 & 5 \\ 7 & 6 \\ 8 & 7 \\ \infty & \end{array}$

$c$
0.88608
0.69374
0.67006
0.65980
0.65455
0.65140
0.64933
$\simeq 0.6412$

Lattice Animals, geometry C

$\begin{array}{rcc}n & n-1 & x_{\mathrm{c}} \\ 2 & 1 & 0.205569431 \\ 3 & 2 & 0.203557211 \\ 4 & 3 & 0.195670026 \\ 5 & 4 & 0.193749993 \\ 6 & 5 & 0.193209253 \\ 7 & 6 & 0.193029170 \\ 8 & 7 & 0.192961633 \\ 9 & 8 & 0.192934795 \\ 10 & 9 & 0.192924195 \\ \infty & & \simeq 0.19292\end{array}$

$\begin{array}{lr}n / \pi \xi_{n} & S_{n} \\ 0.306349 & 2 \\ 0.648407 & 3 \\ 0.677722 & 6 \\ 0.693079 & 9 \\ 0.694011 & 19 \\ 0.692584 & 31 \\ 0.690904 & 67 \\ 0.689450 & 129 \\ 0.688258 & 290 \\ 0.687287 & 623 \\ \simeq 0.685 & \end{array}$

$S_{n}$

2

6

9

19

31

29

90

23

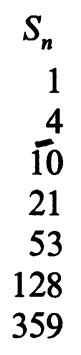

$\simeq 0.677$ fits the whole sequence best. The procedure [3] was the following : for example to estimate $T_{c}$, choose a value of $T_{\mathrm{c}}$ and plot $\log \left(T_{\mathrm{c}}^{(n)}-T_{\mathrm{c}}\right)$ versus $\log n$. The value of $T_{\mathrm{c}}$ and the error bars were estimated by looking at the range of $T_{\mathrm{c}}$ for which the curve has no strong curvature.

The problem with this method is that it gives equal importance to all sizes $n$ instead of giving more weight to the larger widths for which equations $(5,6)$ are expected to be valid. Thus, following Herrmann and Stauffer [5], we use here a modification where an effective correction exponent $\omega_{n}$ is determined from each triplet $v_{n-1}, v_{n}, v_{n+1}$ of consecutive estimates for $v$ obtained by solving $(3,4)$ (with analogous methods applied to the estimates $\left.T_{\mathrm{c}}^{(n)}\right)$ :

$$
\frac{v_{n-1}-v_{n}}{v_{n}-v_{n+1}}=\frac{(n-1)^{-\omega_{n}}-n^{-\omega_{n}}}{n^{-\omega_{n}}-(n+1)^{-\omega_{n}}}
$$

$\begin{array}{lr}n / \pi \xi_{n} & S_{n} \\ 1.007114 & 1 \\ 0.803781 & 4 \\ 0.738495 & 10 \\ 0.718537 & 21 \\ 0.708116 & 53 \\ 0.701775 & 128 \\ 0.697457 & 359 \\ \simeq 0.677 & \end{array}$

$\begin{array}{lr}n / \pi \xi_{n} & S_{n} \\ 0.581457 & 1 \\ 0.589475 & 2 \\ 0.645865 & 4 \\ 0.667477 & 6 \\ 0.676074 & 12 \\ 0.679869 & 19 \\ 0.681676 & 38 \\ 0.682560 & 70 \\ 0.682979 & 145 \\ \simeq 0.683 & \end{array}$

With a programmable hand calculator one can easily determine $\omega_{n}$ from this non-linear equation, provided $n$ is large enough such that $v_{n-1}-v_{n}$ has the same sign as and is larger than $v_{n}-v_{n+1}$. Once $\omega_{n}$ is determined, one can fit a straight line exactly through the plot of $v_{n}$ versus $n^{-\omega}$, using only the three points $n-1, n$ and $n+1$. The intercept then gives another and usually much better estimate for $v$; but again this estimate depends slightly on $n$. We will give below our results calculated in this way from our « raw data " in the table I.

This first method has the disadvantage that the correction analysis for $v_{n}$ is completely decoupled from that of $T_{c}^{(n)}$; thus the resulting estimates for the correction exponents differ for $v_{n}$ and for $T_{c}^{(n)}$. In reality, only one leading correction term is expected to describe the asymptotic convergence. To avoid that theoretical drawback we therefore developed a second 
method which incorporates the corrections from the beginning instead of the above $a$ posteriori method. This $a$ posteriori method is in the same spirit as other extrapolation techniques $[15,16]$ which have been used to analyse the results of the phenomenological renormalization. Even more accurate might be, at least for some of our data, the Romberg type extrapolation of Beleznay [17].

For this purpose we assume that instead of the leading behaviour in equation (1) with correlation length $\xi_{n}$ simply proportional to $n$ at $T=T_{c}$, we have :

$$
1 / \xi_{n}\left(T_{c}^{(n)}\right)=a / n+b / n^{1+\omega}+\cdots .
$$

In the same spirit as for our first method we allow the correction exponent $\omega$ in equation (8) to depend on $n$ in order to take into account the higher order corrections neglected in equation (8). Since now $\omega$ and $T_{c}$ need to be calculated simultaneously, we need two coupled equations to solve, based on four consecutive strip widths :

$\frac{n \xi_{n}^{-1}\left(T_{\mathrm{c}}\right)-(n-1) \xi_{n-1}^{-1}\left(T_{\mathrm{c}}\right)}{n \xi_{n}^{-1}\left(T_{\mathrm{c}}\right)-(n-2) \xi_{n-2}^{-1}\left(T_{\mathrm{c}}\right)}=\frac{n^{-\omega}-(n-1)^{-\omega}}{n^{-\omega}-(n-2)^{-\omega}}$

$$
\begin{aligned}
& \frac{(n+1) \xi_{n+1}^{-1}\left(T_{\mathrm{c}}\right)-n \xi_{n}^{-1}\left(T_{\mathrm{c}}\right)}{(n+1) \xi_{n+1}^{-1}\left(T_{\mathrm{c}}\right)-(n-1) \xi_{n-1}^{-1}\left(T_{\mathrm{c}}\right)}= \\
& =\frac{(n+1)^{-\omega}-n^{-\omega}}{(n+1)^{-\omega}-(n-1)^{-\omega}} \text {. }
\end{aligned}
$$

Again, these two non-linear equations for $\omega$ and $T_{\mathrm{c}}$ have a solution only for large enough $n$ when the initial fluctuations for small $n$ have died out; and again the resulting values $\omega$ and $T_{\mathrm{c}}$ depend slightly on $n$. In our later figures we associate the results from this a priori method of equation (9) with the average index $n-1 / 2$; therefore we gave no index to $\omega$ and $T_{\mathrm{c}}$ in equation (9) since otherwise the notation would be too complicated.

The main idea in this a priori method consists in using from the beginning several strip widths to determine the correction to scaling exponent $\omega$. This idea has been already proposed by Barber [18] in the study of the Ising model.

Before we present our results for these two methods, the $a$ posteriori equation (7) and the $a$ priori equation (9), we shortly list the problems to which we applied them.

\section{The models.}

This paper considers in three different geometries two models already studied by Derrida and de Seze [3] : site percolation and lattice animals [13]. For site percolation, every lattice site is randomly occupied with probability $p$, and clusters are groups of occupied neighbouring sites. The probability that two sites at distance $r$ apart belong to the same cluster varies asymptotically as $\exp (-r / \xi)$, with the connectivity length $\xi$ diverging as $\left(p_{c}-p\right)^{-v}$ if the concentration approaches the percolation threshold $p_{\mathrm{c}}$ from below. This threshold plays the role of $T_{c}$ and $v$ is $4 / 3$ in two dimensions. The number $g_{s}$ of lattice animals is the number of different cluster configurations one can build with $s$ sites constrained to form one cluster. The generating function $\Sigma_{s} g_{s} x^{s}$ diverges at some critical point $x=x_{\mathrm{c}}$ since $g_{s}$ increases exponentially with $s$ :

$$
\log \left(1 / x_{\mathrm{c}}\right)=\lim _{s \rightarrow \infty}\left(\frac{1}{s} \log g_{s}\right) .
$$

Thus $x_{\mathrm{c}}$ here plays the role of $T_{\mathrm{c}}$. The average radius of gyration for the lattice animals increases for large cluster size $s$ as $s^{v}$, which defines our correlation length exponent, in analogy to self-avoiding walks and similar objects. In contrast to percolation, we may also call $1 / v$ the fractal dimension for the lattice animals; and no exact solution is known for any animal $x_{c}$ or $v$ in two-dimensions.

We looked at three geometries denoted by the letters A, B and C here :

A) square lattice with transfer direction in the direction of the axis.

B) square lattice with transfer direction in the diagonal;

C) triangular lattice with transfer direction following a lattice axis. In all cases, periodic boundary conditions were imposed across the strips.

Our tables give the raw data used for the a posteriori analysis as well as the end results of our a priori analysis, as a function of strip width $n$. The $n=\infty$ result for the a posteriori tables is our extrapolation based on these data alone, or is the exactly known result. The ratio $n / \pi \xi_{n}$ gives asymptotically [7] the exponent $\eta=2-\gamma / v$ in percolation; its significance for lattice animals still needs to be clarified.

For example, the $a$ posteriori evaluation of lattice animals with geometry A up $n=11$, used about one hour on a Cray vector computer. Computer memory, not time, was the limiting problem. Our computer time is appreciably smaller than that used for a typical Monte Carlo experiment with which will compare our results. (In contrast to Monte Carlo studies, our results cannot be improved by repeating the runs since they are, we hope, exact and not based on random processes). As usual in this type of studies as well as in series expansions, most of the computer time in each problem was spent on the largest width, not on all smaller widths together.

\section{Analysis.}

The reader may use himself the data in our tables to find out the desired critical points $\left(p_{\mathrm{c}}\right.$ or $\left.x_{\mathrm{c}}\right)$ and exponents $(v)$ for $n=\infty$. We present in our figures 
Table II. - Extrapolated critical points and exponent from a priori analysis, equation (9). $a, b$ and $\omega$ refer to equation (8).

Percolation, geometry $A$

$\begin{array}{rcccc}n & p_{\mathrm{c}} & \omega & a & b \\ 7,6,5,4 & 0.59340079 & 0.69337 & 0.2027175 & 0.023026 \\ 9,8,7,6 & 0.59305797 & 1.02569 & 0.2060444 & 0.025362 \\ 10,9,8,7 & 0.59291350 & 1.19792 & 0.207520 & 0.028320\end{array}$

Percolation, geometry $B$

$\begin{array}{cc}{ }^{n} & p_{\mathrm{c}} \\ 5,4,3,2 & 0.59104798 \\ 6,5,4,3 & 0.59234716 \\ 7,6,5,4 & 0.59259074 \\ 8,7,6,5 & 0.59268233\end{array}$

$\omega$

4.0555686

2.7529618

2.4067940

2.2101754

Lattice Animals, geometry $B$

${ }^{n}$
$5,4,3,2$
$6,5,4,3$
$7,6,5,4$
$8,7,6,5$
Lattice Animals, geometry $C$

$x_{\mathrm{c}}$
0.24580739
0.24611946
0.24614246
0.24614870

$\omega$

2.60417

1.68963

1.58472

1.53627

$a$
0.214084165
0.210121710
0.209166659
0.208735445

$a$

0.698604

0.683411

0.681472

0.680741 b

0.31483637

0.11316710

0.08319056

0.06819344 $\omega$

1.89129

2.78830

3.23699

3.62355

4.19755

5.1754 $a$

0.700288

0.686971

0.684981

0.684270

0.683813

0.683508

$b$
0.35194
0.20541
0.19176
0.18438

only those examples where the convergence was best, ignoring those sequences with oscillations or extrema even for large $n$. We found that none of three geometries A, B and C is clearly preferable to the others in all cases; nor did the new a priori method, equations(9), necessarily give better or worse results than the more conventional $a$ posteriori analysis, equation (7).

For all figures, the symbols $\square, \times$ and $\nabla$ refer to geometries A, B and C with the $a$ priori analysis of equation (9) and merely repeat the numbers given in the appropriate table; for the a posteriori analysis of the data given in the other tables we show our results in the figures by the symbols $\diamond,+$, and $\Delta$ for geometries $\mathrm{A}, \mathrm{B}$ and $\mathrm{C}$.

Figure 1 gives $p_{\mathrm{c}}=0.49999$ (7) and 0.59274 (10) for the percolation thresholds in the triangular and square lattice, respectively. The number in parenthesis gives our subjectively estimated error bars for the last digit(s) shown, e.g. $p_{\mathrm{c}}=0.59274 \pm 0.00010$. Our results are compatible with the exact $p_{\mathrm{c}}=1 / 2$ for the triangular lattice and with the Monte Carlo estimates 0.59270 (10) of Rapaport and 0.59277 (5) Gebele [8]. If we would assume that the extrapolated data for larger unknown $n$ vary monotonically we could make our error bars about half as large. It is quite satisfactory that here as well as in our later examples one has some data approaching the estimated limit from above and others approaching it from below; for any single set of data therefore we would be less confident of our final estimate than from our combination of all suitable results.

Our data here and later are plotted versus $n^{-4}$ since this method gave smooth curves and a suitable separation of data and limits. We do not assert to have shown that the second correction term indeed varies as $n^{-4}$ asymptotically.

For the critical fugacities $x_{\mathrm{c}}$ of lattice animals in the triangular and square lattice, figure 2 suggests 0.192925 (10) and $0.246150(10)$, the latter result being fully comparable with 0.246148 (12) from direct animal counts using ten months of PDP 11/70 computer time [9], and five times more accurate than the identical estimate from our earlier transfer matrix approach [3]. (No recent accurate determinations for the triangular lattice are known to us.)

Figure 3 gives our nicest result, $v=0.64075$ (15) for the animal radius exponent, one of the most accurate exponents ever determined numerically. The data of our a posteriori analysis for geometry A show 


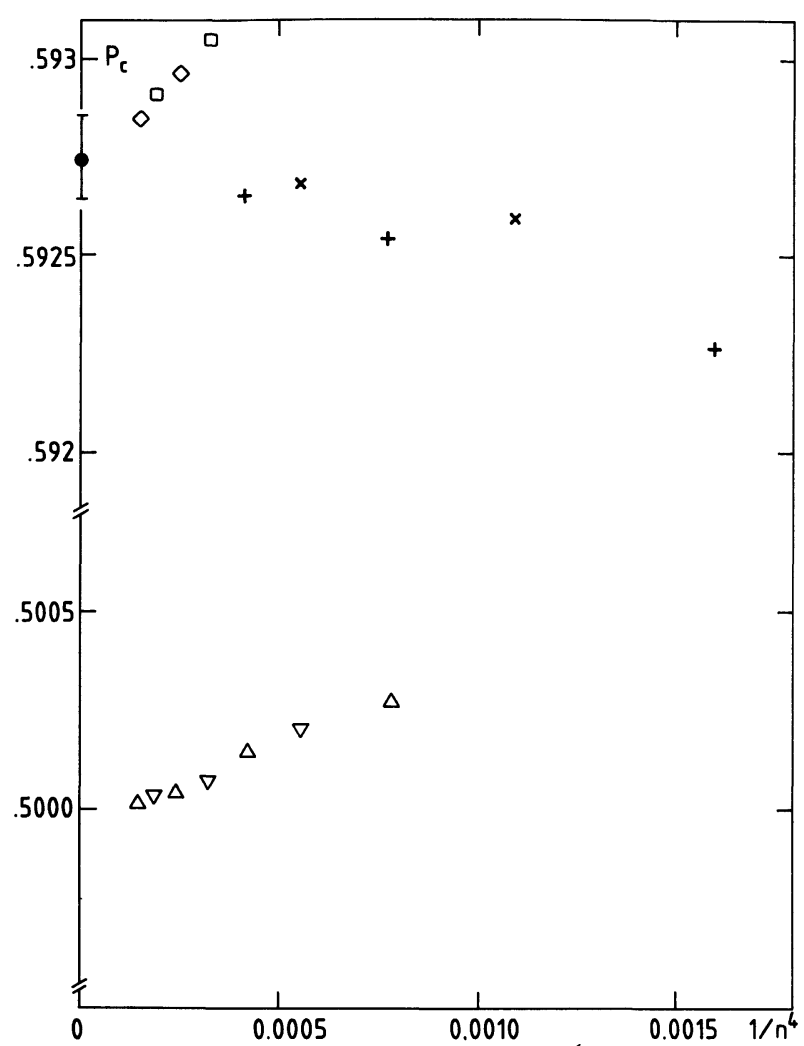

Fig. 1. - Results for the two-dimensional percolation threshold. The intercepts here and in figures 2-4 give the asymptotic results, as indicated by our error bars. The symbols have the same meaning and are explained in the text, for all 4 figures.

a minimum near $n=10$. Our estimate agrees with [3] but has half as large error bars.

The upper part of figure 3 shows that our a priori estimates for the correction exponent $\omega$ are orders of magnitude worse than those for the leading quantities. Nevertheless $\omega=1.8 \pm 0.3$ seems justified, confirming the less accurate analysis in reference [5] and compatible with $\omega=2$ for percolation [10].

We were less lucky with the exactly known percolation exponents $v=4 / 3$ and $\eta=15 / 72=0.2083333$. For $\eta$ our data, as shown in figure 4 , still vary quite strongly with $n$ and allow only the estimate 0.2088 (8), whereas for $v$ only one curve (geometry $\mathrm{C}$, a posteriori analysis) gave fair results near 1.334 for $n$ near 6 to 9 .

Finally, the quantity $n / \pi \xi_{n}$ approaches about 0.68 for lattice animals. Although this ratio seems universal [14] (see our tables for the three geometries) it is not clear to us how it is related to the exponent $\eta$ or to other known exponents of lattice animals. We just noticed empirically that 0.68 is very close to $(1-v / 2)$.

Not shown in our figures are the tabulated results for $v, a_{1}$ and $b_{1}$ determined a priori form differentiating the correlation length :

$$
n \frac{\mathrm{d}}{\mathrm{d} p} \xi_{n}^{-1}=n^{1 / v}\left(a_{1}+b_{1}^{-\omega}\right)
$$

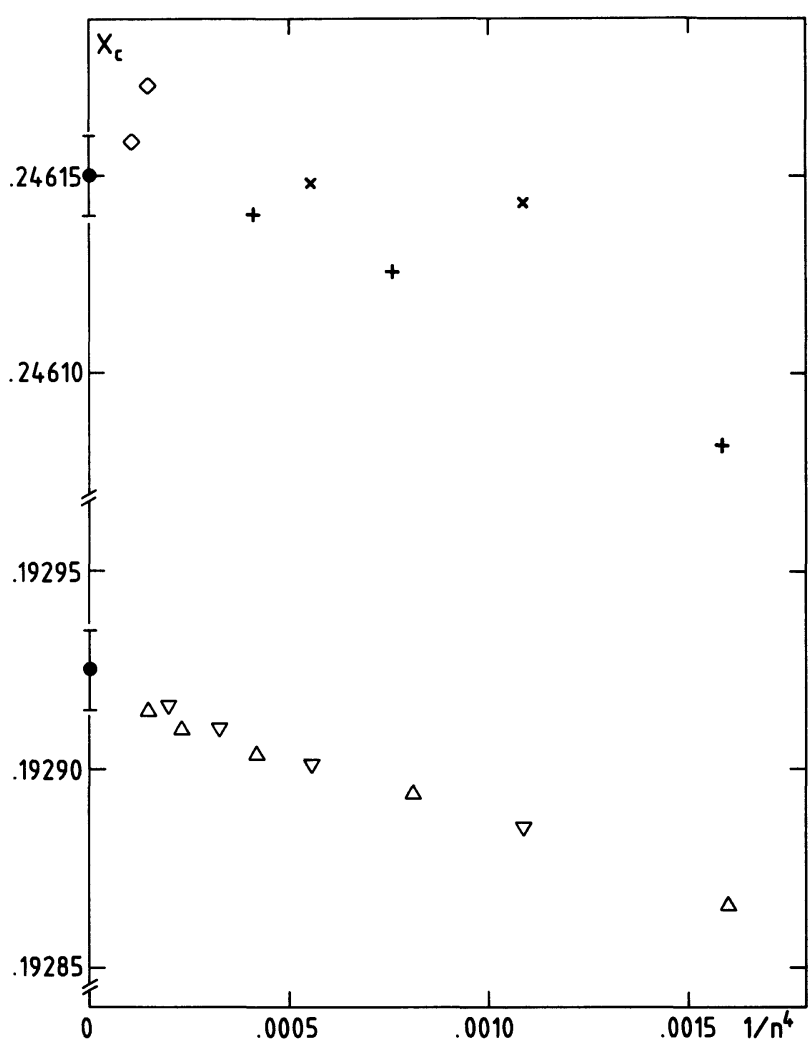

Fig. 2. - Result for the critical point (fugacity, inverse growth factor) for lattice animals in the triangular and square lattice.

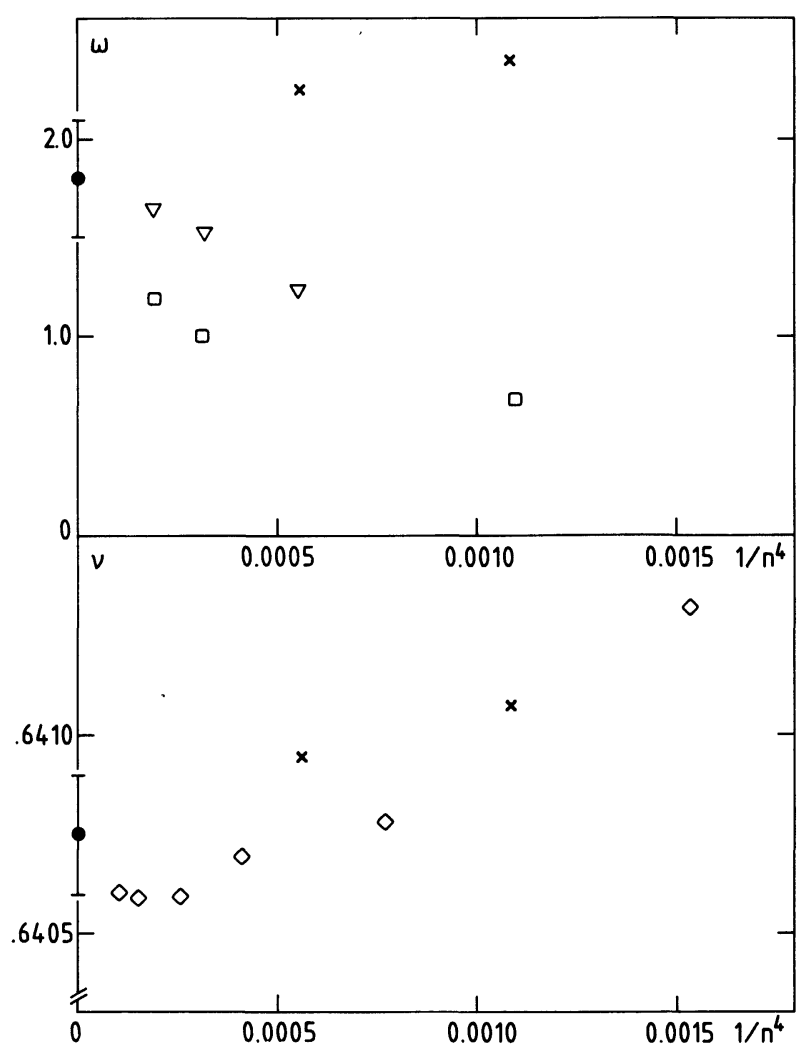

Fig. 3. - Results for the animal radius exponent (bottom) and the percolation correction exponent (top). 


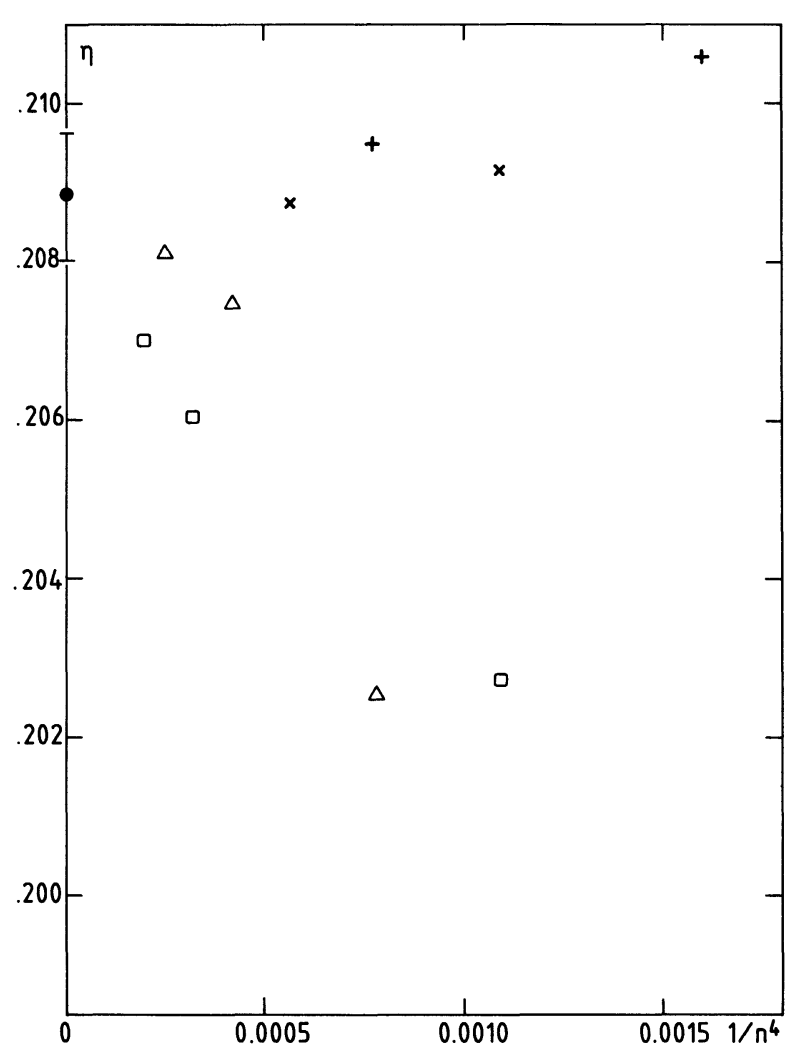

Fig. 4. - Results for the percolation exponent $\eta=n / \pi \xi_{n}$.

with parameters fitted from three consecutive values for $n$. (For lattice animals, $\mathrm{d} / \mathrm{d} p$ is replaced by $\mathrm{d} / \mathrm{d} x$, of course). The derivative was obtained directly from the transfer matrix, and not by inaccurate numerical differentiation of $1 / \xi_{n}$. The reader may check himself that these independent data confirm the results in the figure 3 for the animal $v$; similar results are obtained if one differentiates $\xi$ instead of $1 / \xi$ (not shown).

\section{Conclusion.}

The present paper was concerned with both methods and results. We feel that for a given amount of computer time the transfert matrix approach for our twodimensional geometric problems is presently superior to series expansion and Monte Carlo simulation, as far as accuracy is concerned. Its disadvantage is the exponentially growing memory required (two matrices of size $S_{n} \times S_{n}$, with $S_{n}$ listed is table I).

We have also shown that the a priori method of incorporating corrections to scaling is a useful alternative, though not necessarily better, than the $a$ posteriori analysis; the combination of these two approaches, equations $(7,9)$, gives more information about possible systematic trends than each method alone.

Our numerical results show that the error bars of
Table III. - Same as table II, but using equation (11).

Percolation, geometry A

$\begin{array}{rccc}n & v & a_{1} & \\ 7,6,5,4 & 1.2277 & -1.2927 & -0.8027 \\ 9,8,7,6 & 1.3060 & -1.5814-0.5412 \\ 10,9,8,7 & 1.3183 & -1.6278-0.5344\end{array}$

Percolation, geometry $B$

\begin{tabular}{|c|c|c|c|c|}
\hline$n$ & $v$ & $a_{1}$ & & $b_{1}$ \\
\hline$, 4,3$ & 1.34213 & -1.2504 & - & 1.631 \\
\hline 54 & 1.34019 & -2.2184 & - & 0.745 \\
\hline 5 & 1.33777 & -2.2053 & - & 0.631 \\
\hline $7,6,5$ & 1.33644 & 2.198 & - & 0.562 \\
\hline
\end{tabular}

Lattice Animals, geometry B

$\begin{array}{ccccl}n & v & a_{1} & & b_{1} \\ 5,4,3,2 & 0.64917 & -5.850 & - & 2.435 \\ 6,5,4,3 & 0.64172 & -5.609 & - & 1.639 \\ 7,6,5,4 & 0.64108 & -5.583 & - & 1.534 \\ 8,7,6,5 & 0.64094 & -5.576 & - & 1.466\end{array}$

Lattice Animals, geometry C

$\begin{array}{rcccc}n & v & a_{1} & & b_{1} \\ 5,4,3,2 & 0.63526 & -3.892 & - & 1.793 \\ 6,5,4,3 & 0.64254 & -4.055 & - & 2.936 \\ 7,6,5,4 & 0.64312 & -4.070 & - & 4.912 \\ 8,7,6,5 & 0.64307 & -4.070 & - & 9.055 \\ 9,8,7,6 & 0.64298 & -4.069 & -25.456 \\ 10,9,8,7 & 0.64289 & -3.523 & -150.593\end{array}$

earlier precision analyses $[3,8,9]$ were reliable, with our actual deviations always being smaller than the earlier published error bar. We hope that the same will be said later about the present estimates.

Our value for the animal length exponent, $v \simeq 0.64075$, confirms that, in contrast to threedimensions, the simple result $v=0.625$ from a Florytype theory [11] is not correct; on the other hand our rough analysis for the leading independent correction exponent $\omega$ is compatible with the theoretical possibility $\omega=2$ for two-dimensional percolation [10].

The present approach of exact transfer matrices cannot be expected to work well in the three dimensions when $n^{2}$ is restricted to be about as large as $n$ in our present two-dimensional study and therefore even $n=4$ would be hard to reach. Instead, a combination of transfer matrix and Monte Carlo methods seems useful for this case [12].

\section{Acknowledgments.}

We thank J. L. Cardy. D. C. Rapaport and M. F. Sykes for advance information of their work, and $\mathrm{H}$. J. Herrmann for a critical discussion of our error bars. 


\section{References}

[1] Nightingale, M. P., J. Appl. Phys. 53 (1982) 7927 ; Vannimenus, J. and Nadal, J. P., Phys. Rep. 103 (1984) 47.

[2] BARber, M. N., Phase Transitions and Critical Phenomena edited by C. Domb and J. L. Lebowitz, 8 (Academic Press, New York) 1984.

[3] Derrida, B. and De Seze, L., J. Physique 43 (1982) 475.

[4] Privman, V. and Fisher, M. E., J. Phys. A 16 (1983) L295.

[5] Herrmann, H. J. and Stauffer, D., Phys. Lett. A 100 (1984) 366

[6] LuCK, J. M., Phys. Rev. B 31 (1985) 3069.

[7] CARDY, J. L., J. Phys. A 17, L385 (1984);

Burkhardt, T. W. and Guim, I., J. Phys. $A$ 18, L25 (1985); $A 18$ (1985) L33 and references therein.

[8] Gebele, T., J. Phys. A 17 (1984) L51 ;

RAPAPORT, D. C., J. Phys. A 18 (1985) L175.
[9] Guttmann, A. J., J. Phys. A 15 (1982) 1987.

[10] Nienhuis, B., J. Phys. A 15 (1982) 199.

[11] IsaAcson, J. and Lubensky, T. C., J. Physique 41 (1980) L469;

De Gennes, P. G., C.R. Hebd. Séan. Acad. Sci. 291 (1980) 17

[12] Saleur, H. and Derrida, B., J. Physique 46(1985) 1043.

[13] Stauffer, D., Introduction to Percolation Theory (Taylor and Francis, London) 1985.

[14] CARDY, J. L., private communication.

[15] Hanver, C. J. and Barber, M. N., J. Phys. A 14 (1981) 2009.

[16] Blöte, H. W. J. and Nightingale, M. P., Physica A 112 (1982) 406.

[17] BELEZNAY, preprint 1985 ;

KERTÉSZ, J., private communication.

[18] BARber, M. N., Phys. Rev. B 27 (1983) 5879. 